\title{
IN-CIRCUIT MEASUREMENT OF COMPLEX CIRCUITS' PARAMETERS WITH ELECTRICAL SEPARATION BY ITERATION METHOD
}

\author{
Alexander Roik \\ Vinnitsya State Technical University, \\ Chmelnitske shose, 95, 286021, Ukraine, Vinnitsya \\ roik@vstu.vinnica.ua \\ Vladimir Mesyura \\ Vinnitsya State Technical University, \\ Chmelnitske shose, 95, 286021, Ukraine, Vinnitsya \\ vimes@vstu.vinnica.ua
}

\begin{abstract}
In a paper the use of iterative methods in the tasks of elements parameters measurement in structure of multi-terminal electrical circuits is considered. In the correspondence with it the iterative processes of an artificial partition of the closed circuits on dipoles are investigated. Thus the generalized equation of an electrical partition of the closed circuits generalized algorithm of an iterative partition and appropriate structure for it of a realization is obtained. The task of iterative processes raise speed are considered also.
\end{abstract}

\section{Statement of the task}

During in-circuit testing of radio-electronic products one of the main problems is measuring of passive two-terminals within multi-terminals electrical circuits. This problem is solved by use of methods of artificial electrical separation of multi-terminal circuit on independent twoterminals.

At present the electrical separation of measured two-terminals from other elements of multiterminal circuit is executed with help of closed structures with balancing in a monitoring mode. As a device for balancing, in this case, operating amplifiers (OA) are used, which in steady state at a reasonably high gain with high accuracy support on inputs identical potentials [1] However, imperfect of the OA characteristics, in conditions of shunting of its inputs, are main sources of measurement methodical errors. Besides, at strong shunting of OA inputs by elements of multi-terminal circuit not included in measurement, especially if they are nonpassive, such structures of measuring converters (MC) are frequently unstable, that limits a opportunity of their use. Thus, a depth and reliability of the testing of diagnosing systems as a whole are essentially reduced.

In order to prevent it, the execution of electrical separation of multi-terminal circuits on twoterminals, using idea of organization of a iterative feedback, which at the choice of optimal parameters of algorithms can ensure absolute convergence of balancing process, is offered.

\section{Solution of the task}

The block diagram of a measuring converter, which provides separation of multi-terminal circuits by iterative methods of balancing, is indicated on fig. 1. Here multi-terminal electrical circuit or unit-under-test (UUT) is given in a kind three-terminal closed electrical circuit with measured two-terminal $\dot{Z}_{x}$ between the terminals $h$ and $s$ and shunting two-terminals $\dot{Z}_{h}$ and $\dot{Z}_{s}$, possessing by the common terminal g. Directly on measured two-terminal $\dot{Z}_{x}$ a voltage $v_{T}=v_{0} \sin \omega t$ is connected, which is formed by a source of testing action. Through 
terminal $\mathrm{s}$ in series with two-terminal $\dot{Z}_{x}$ a reference resistor is connected, the second terminal of which is connected to a ground bus.

Multi-terminal electrical circuit (UUT) and reference resistor $R_{0}$ will form a four-terminal, which must be balanced to solve problem of electrical separation. For this purpose on terminal $\mathrm{g}$ of multi-terminal circuit balancing voltage $\varphi(\dot{w})$, where $\dot{w}$ - result of measurement, i.e. MC output signal, and - $\varphi(\dot{w})$ is transformation function.

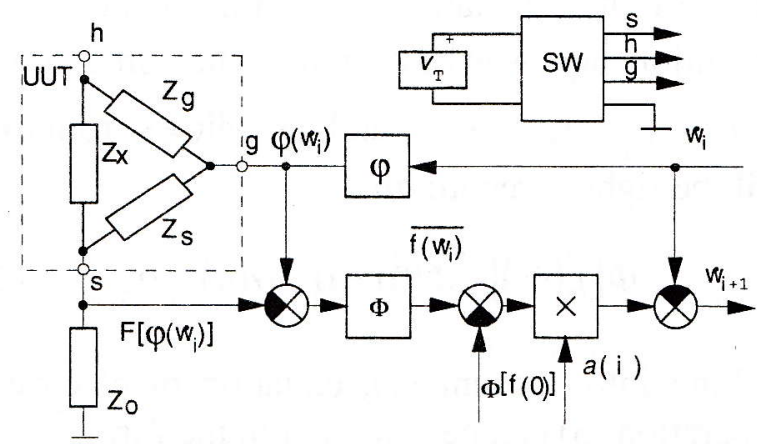

Fig.1 Block diagram of self-correcting MC with electrical separation by iterative method.

We shall receive iterative algorithm of the problem solution of multi-terminal circuit electrical separation. Taking into account above described, in terminal s of multi-terminal circuit a voltage $F[\varphi(\dot{w})]$ will be formed, which is determined by following expressions:

$$
\begin{aligned}
& F=\varphi(\dot{w})(1-\dot{\gamma})+\beta ; \\
& \dot{\gamma}=\dot{Z}_{s} /\left(\dot{Z}_{s}+R_{0}\right) ; \\
& \dot{\beta}=v_{l} \dot{Z}_{s} R_{0} / \dot{Z}_{x}\left(\dot{Z}_{s}+R_{0}\right)=\dot{\gamma} \varphi\left(\dot{Z}_{x}\right) ; \\
& \varphi\left(\dot{Z}_{x}\right)=v_{T} R_{0} / \dot{Z}_{x} .
\end{aligned}
$$

Then, the problem of measured two-terminal electrical separation $\dot{Z}_{x}$ from $\dot{Z}_{s}$ and $\dot{Z}_{h}$ will consist in following, find such value $\dot{w}_{*}$, for which difference of voltages on the terminals $g$ and $\mathrm{s}$ UUT was equaled to zero.

$$
\varphi\left(\dot{w}_{*}\right)-F\left[\varphi\left(\dot{w}_{*}\right)\right]=0 .
$$

Obviously, the current through a branch $\dot{Z}_{s}$ will be also equal for a zero, that it is possible to consider as electrical gap in this branch. In this case, the voltage on the terminal s of UUT will be proportional to parameters of measured twoterminal $\dot{Z}_{x}$ and does not depend from parameters $\dot{Z}_{s}$.

Mathematically, search of $W_{*}$ value, for which (2) is fair, is equation solving:

$$
f(\dot{w}) \equiv(\dot{w})-F[\varphi(\dot{w})]=0 .
$$

We shall name it as a equation of electrical separation.

To solve this equation (3) we will use numerical methods.

For this purpose equation (3) we will reduct to a form $\dot{w}=\psi(\dot{w})$, then, choosing some initial approximation $\dot{w}_{1}, \quad$ calculate consecutive approximations:

$$
\dot{w}_{i+1}=\psi\left(\dot{w}_{i}\right) ; \quad i=\overline{1, n} ; \quad n \rightarrow \infty .
$$

According to the theorem about compressing mappings, if in a complex plane there is such area $\mathrm{W}$ and positive number $v<1$ (compressed mapping), that for all $\dot{w}_{i}, \dot{w}_{j} \in \dot{W}$ condition is satisfied: $\left.\quad d \mid \psi\left(\dot{w}_{i}\right), \psi\left(\dot{w}_{j}\right)\right\rfloor \leq v d\left(w_{i}, w_{j}\right) ; i \neq j$, then set $\dot{W}$ contains a unique stationary point $\dot{w}_{*}$ of mapping $\psi$, for which $\dot{w}_{*}=\psi\left(\dot{w}_{*}\right)$, and, $\dot{w}_{*}$ is a limit of any sequence of points, determined from (4) for any initial point $\dot{w}_{1} \in W$. The speed of decrease of a absolute error of a approaching sequence after one and after $\mathrm{n}$ iterations can be evaluated accordingly formulas:

$$
\begin{aligned}
& d\left(\dot{w}_{n}, \dot{w}_{*}\right) \leq v d\left(\dot{w}_{n-1}, \dot{w}_{*}\right) \\
& d\left(\dot{w}_{n}, \dot{w}_{*}\right) \leq v^{n} d\left(\dot{w}_{1}, \dot{w}_{*}\right) .
\end{aligned}
$$

Obviously, that for relative errors of a approaching sequence following expressions will be also fair: 


$$
\begin{aligned}
& \delta_{n} \leq v \delta_{n-1} ; \quad \delta_{n} \leq v^{n} \delta_{1} ; \\
& \delta_{(\cdot)}=d\left(\dot{w}_{(\cdot)}, \dot{w}_{*}\right) / \dot{w}_{*} .
\end{aligned}
$$

Equation (3) we will reduct to a form (4) as follows. We will subject both parts (3) under action of operator $\boldsymbol{\Phi}$, which in our case represents function of phase-sensitive transformation of a real and imagine parts of signal into some, proportional to them, constant values, determined as

$$
\begin{aligned}
& \Phi(\cdot)=\left\{\Phi_{R}(\cdot), \Phi_{I}(\cdot)\right\} ; \\
& \Phi_{R}(\cdot)=\operatorname{Re}\left[(\cdot)\left(1+\dot{\gamma}_{\phi}\right)+\dot{\beta}_{\phi}\right] ; \\
& \Phi_{I}(\cdot)=\operatorname{Im}\left[(\cdot)\left(1+\dot{\gamma}_{\phi}\right)+\dot{\beta}_{\phi}\right],
\end{aligned}
$$

where $\dot{\gamma}_{\Phi}$ and $\dot{\beta}_{\Phi}$ - accordingly a multiplicative and additive part of error of phase-sensitive transformation.

Then the operator $\Phi(0)$ from a right part of obtained expression will transfer in left-hand i.e. $\Phi[f(\dot{w})]-\Phi(0)=0$, multiplicative both its part on some multiplier $(-\alpha)$ and add to both parts variable $\dot{w}$. In result we will have a equation of electrical separation in form:

$$
\dot{w}=\dot{w}-\alpha\{\Phi[f(\dot{w})]-\Phi(0)\}
$$

The solution of a equation (9), according to (4), can be realized on algorithm

$$
w_{n+1}=w_{n}-\alpha(n)\left\{\Phi\left[f\left(\dot{w}_{n}\right)\right]-(0)\right\},
$$

where multiplier $\alpha(n)$ - parameter of algorithm, named as a step.

Obtained algorithm we will name as generalized algorithm of electrical separation of multiterminal circuits by method of iterations. Process of electrical separation on each iteration of algorithm (6) is executed in three stages, first two of which are measuring, and third computing. The first stage - determination of $\Phi$ $(0)$, initial data for which are values $v_{T}=0$ and $w=0$. Then, received value of $\boldsymbol{\Phi}(0)$ is stored in a managing computer. At the second stage values $\Phi\left[f\left(\dot{w}_{i}\right)\right]$ are determined at action of a testing signal $v_{T}=v_{0} \sin \omega t$. At the third stage calculation of $(i+1)$-th approximation $w_{i+1}$ is executed, which according to algorithm (6) consists in reception of difference $\Phi\left[f\left(\dot{w}_{i}\right)\right]-\Phi(0)$ by its multiplication on a step of algorithm $\alpha(i)$ and subtraction of received result from the previous approximation $\dot{w}_{i}$. If algorithm (6) converges, then cyclic fulfillment of described above measuring-computing operations, at sufficient $\mathrm{n}$, for some, positive and no matter how small beforehand given value of absolute deviation $\mathcal{E}$, fulfillment of a condition $\left|\dot{w}_{n+1}-\dot{w}_{*}\right| \leq \varepsilon$ will ensure. In a limit, at $n \rightarrow \infty, \dot{w}_{n+1} \rightarrow \dot{w}_{*}$, and, as follows from (6), will be right to record, that

$$
\Phi\left[f\left(\dot{w}_{i}\right)\right]-\Phi(0)=0 ; \alpha(n) \neq 0 .
$$

Taking into account (1), equation of electrical separation (3) can be rewritten in the form:

$$
f(\dot{w})=\dot{\gamma}\left[v_{0} R_{0} \dot{Y}_{x} \sin \omega t-\varphi(\dot{w})\right] .
$$

Here we will determine function of a reverse converter according to expression:

$$
\varphi(\dot{w})=\dot{\beta}_{x}+(1+\dot{\gamma}), \dot{w}_{*} \sin \omega t,
$$

where $\dot{\gamma}_{\varphi}$ and $\dot{\beta}_{\varphi}$ - multiplicative and additive parts of error of a reverse converter. Then, substituting (8) and (9) in the equation (7) and taking into account (5) we will receive:

$$
\begin{aligned}
& \Phi\left[f\left(\dot{w}_{*}\right)\right]=\{\operatorname{Re}[\overline{f(\dot{w})}\rfloor+j \operatorname{Im}[\overline{f(\dot{w})}]\} \\
& \overline{f(\dot{w})}=\dot{\gamma}\left(1+\dot{\gamma}_{\phi}\right)\left[v_{0} R_{0} \dot{Y}_{x}\left(1+\dot{\gamma}_{\phi}\right) \dot{w}_{*}\right]+\dot{\beta}_{\Sigma} ; \\
& \dot{\beta}_{\Sigma}=\dot{\beta}_{\Phi}-\left(1+\dot{\gamma}_{\phi}\right) \dot{\beta}_{x} .
\end{aligned}
$$

It is visible, that at values $v_{0}=0$ and $\dot{w}_{*}=0$ in the equation (7) the additive part of error will be determined as $\Phi(0)=\dot{\beta}_{\Sigma}$. Hence, if to consider, that the values $\dot{\gamma}$ and $\left(1+\dot{\gamma}_{\Phi}\right)$ are not equal to a zero, solution of the equation of electrical separation can be presented in the form:

$$
\dot{w}_{*}=v_{0} R_{0} \dot{Y}_{x} /\left(1+\dot{\gamma}_{\varphi}\right) \text {. }
$$


If to designate, that $\dot{\xi}_{\varphi}=-\dot{\gamma}_{\varphi} /\left(1+\gamma_{\varphi}\right)$, then the equality (11) will be rewrite as:

$$
\dot{w}_{*}=v_{0} R_{0} \dot{Y}_{x}\left(1+\xi_{\varphi}\right)
$$

When real and imagine parts extracting in the (12) we will receive relations, connecting results of measurements $w_{*}^{R}$ and $w_{*}^{I}$ with parameters measured two-terminal $Y_{x}^{R}$ and $Y_{x}^{I}$ :

$$
\begin{aligned}
& w_{*}^{R}=v_{0} R_{0}\left[Y_{x}^{R}\left(1+\xi_{\varphi}^{R}\right)-\xi_{\varphi}^{l} Y_{x}^{I}\right] \\
& w_{*}^{I}=v_{0} R_{0}\left[Y_{x}^{I}\left(1+\xi_{\varphi}^{R}\right)-\xi_{\varphi}^{I} Y_{x}^{R}\right] .
\end{aligned}
$$

Thus, in case of convergence of algorithm (6), till his ending balance of a circuit is reached. Thus, three problems are solved simultaneously, namely: mode of electrical break in the branch $\dot{Z}_{s}$ is provided, errors of transformation $\dot{\gamma}_{\Phi}$ and $\dot{\beta}_{\Phi}$ are corrected and measurement of parameters of considered twoterminal is executed. Restrictions on accuracy for such $\mathrm{MC}$ are accuracy of the given voltage $v_{0}$ and reference element $R_{0}$, as well as multiplicative error of a reverse converter $\dot{\xi}_{\varphi}$.

The first two restrictions are not essential, as far as from the technical point of view it is enough easy to ensure ignorable small errors of voltage source and reference resistor. Hence, main metrological limiting factor of accuracy maintenance of measurement are reverse converter errors.

On the other hand from the theory of measurements it is known, that extreme achievable accuracy is limited only to errors of a multiple-valued measure. In this connection, research was conducted, the results of which have allowed to develop a method and algorithm of correction of a RC error $\xi$, concluded in a combination of above considered algorithm and method of measured values replacement [2].
As a replacing values it is conveniently to choose testing action $v_{0} \sin \omega t$. Then, using for it similar to (6) algorithm measuring, we will have:

$$
\left.w_{k+1}^{v}=w_{k}^{v}-\alpha(k)\left\{\Phi \mid \varphi\left(w_{k}^{v}\right)-v_{T}\right\rfloor-\Phi[\varphi(0)-0]\right\} \text {. }
$$

The approximation $w_{k+1}^{v} \rightarrow w_{*}^{v}$ with measured value $v_{0}$ will be connected by analogy with relation (12)

$$
\dot{w}_{*}^{v}=v_{0}\left(1+\dot{\xi}_{\varphi}\right) .
$$

From (12) and (14) obviously, that having divided $\dot{w}_{*}^{v}$ from (14) on $\dot{w}_{*}^{z}$ from (12), final result of measurement:

$$
\dot{w}_{x x}=\dot{w}_{*}^{v} / \dot{w}_{*}^{z}=\dot{z}_{x} / R_{0}
$$

will be free from all parts of errors as direct as return ways of transformation.

Thus, if in (15) to substitute expressions for $w_{*}^{z}$ and $w_{*}^{v}$ we will receive algorithm:

$$
\dot{w}_{* *}=\frac{\dot{w}_{k}^{v}-\alpha(k)\left\{\Phi\left[\varphi\left(w_{k}^{v}\right)-v_{T}\right]-\Phi[\varphi(0)-0]\right\}}{\dot{w}_{n}^{z}-\alpha(n)\left\{\Phi\left[\varphi\left(\dot{w}_{n}^{2}\right)\right]-\Phi[f(0)]\right\}}
$$

Which we will name as generalized self-corrected algorithm of electrical separation of UUT by method of iteration. On fig.1 the switch SW at measuring of $\dot{Z}_{x}$ connect $v_{T}$ directly to $\dot{Z}_{x}$, and at measuring $v_{T}$ this signal is connected to element $R_{0}$, and terminal $h$ connected to ground bus.

\section{Conclusions}

Methods and tools offered in paper for measuring of passive components' parameters in multiterminal circuits with usage of iterative algorithm of electrical separation ensure more high accuracy of measurements and convergence of balancing process. Usage of such $\mathrm{MC}$ in in-circuit test equipment will allow to increase its efficiency and credibility during testing radio-electronic products. 


\section{References}

[1] Fault Diagnosis of Analog Circuit/ J.W. Bandler, A.E. Salama. Proc.of IEEE, 1985, vol.73, N.8, p.1279-1325

[2] Bayda N.P., Mesyura B.I., Roik A.M. Self leaning Manufacturing Defect's Analizers for REE.Moscow: Radio and Communications,1991.256p.(in Russian). 